\title{
Molecular Docking, 3D Structure-Based Pharmacophore Modeling, and ADME Prediction of Alpha Mangostin and its Derivatives against Estrogen Receptor Alpha
}

\author{
Muchtaridi Muchtaridi ${ }^{1 *}$, Doni Dermawan ${ }^{1}$, Muhammad Yusuf ${ }^{2}$ \\ Department of Pharmaceutical Analysis and Medicinal Chemistry, Faculty of Pharmacy, Universitas Padjadjaran, INDONESIA. \\ 2Department of Chemistry, Faculty of Mathematics and Natural Sciences, Universitas Padjadjaran, INDONESIA.
}

\begin{abstract}
Objective: The aims of this study are to identify the molecular interactions and the pharmacophore-fit of of $\alpha$ mangostin and its derivatives with estrogen receptor $\alpha(E R \alpha)$ using computational simulation approaches to obtain new potent of anti-breast cancer. Materials and Methods: Molecular docking simulation and 3D structure-based pharmacophore models were employed to identify the molecular interactions of $\alpha$-mangostin and its derivatives against estrogen receptor $\alpha$ (ER $\alpha)$ (PDB ID: 3ERT). Results: The results showed that the binding energy of $\alpha$-mangostin and its best derivative (AMD10) were $-9.05 \mathrm{kcal} / \mathrm{mol}$ and $-11.89 \mathrm{kcal} / \mathrm{mol}$, respectively. These compounds also interacted with Thr347, Asp351, Met388, Met528, Ile424, Arg394, and Glu353. The pharmacophore-fit scores of $\alpha$-mangostin and AMD10 were $83.06 \%$ and $86.46 \%$, respectively. In addition, the absorption, distribution, metabolism and excretion (ADME) properties
\end{abstract}

were predicted. Conclusion: These results showed that $\alpha$-mangostin and AMD10 are promising candidates of novel anti-breast-cancer agents with antagonistic activity to $E R \alpha$.

Key words: $\alpha$-mangostin, Estrogen receptor alpha, Molecular docking, Pharmacophore.

Correspondence

Muchtaridi Muchtaridi, Department of Pharmaceutical Analysis and Medicinal Chemistry, Faculty of Pharmacy, Universitas Padjadjaran, INDONESIA.

Phone: +62-22-84288888 (ext.3510)

Email: muchtaridi@unpad.ac.id

DOI: 10.5530/jyp.2018.10.58

\section{INTRODUCTION}

Cancer is the leading cause of death with the second highest prevalence rate in the world after ischemic heart disease. Total of 8.8 million people die because of cancer by $2015 .{ }^{1}$ Breast cancer is one of the highest prevalence of cancer in Indonesia from 2007 to 2016 with the incidence of 611 cases. West Kalimantan is the province with the highest prevalence of

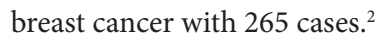

Estrogen receptors are the major prognostic markers used to identify tumors in breast tissue. ${ }^{3}$ The estrogen receptor consists of two subtypes namely ER $\alpha$ and ER $\beta$ that have different affinities to estrogen. The estrogen $\alpha(E R \alpha)$ receptor is an activated ligand by transcriptional regulator which is the main regulator of breast differentiation and proliferation. ${ }^{4}$ The estrogen receptor $\alpha(\mathrm{ER} \alpha)$ plays an important role in the development and progression of dependent hormonal type breast cancer. ${ }^{5}$

Tamoxifen as an anti-estrogen blocks the estrogenic signal through a mechanism of competition with endogenous estrogens to bind to estrogen receptors and modify its activity as a dependent latch of transcriptional regulators. Tamoxifen has antagonistic activity in the breast but it is agonists in the uterus and bone. ${ }^{6}$ Tamoxifen and its active metabolite 4-hydroxytamoxifen (4-OHT) have cytotoxic activity against MCF-7 breast cancer cells with $\mathrm{IC}_{50} 5 \mu \mathrm{M}$ and $1 \mu \mathrm{M}{ }^{7}$ However, the efficacy of tamoxifen is limited by the presence of intrinsic and possible resistance. Excessive amplification and/or expression COPS5 (COP9 complex subunit) is one of the main causes of tamoxifen resistance in $86.7 \%$ of $\mathrm{ER} a+$ breast cancer patients. COPS5 overexpression through isopeptidase activity results in the degradation of proteasome-mediated NCoR which is a key ERCC repressor. ${ }^{8}$

One of the new drug discovery efforts to treat breast cancer is through the use of natural compounds such as a-mangostin compounds that obtained from the mangosteen pericarp. $\alpha$-mangostin as the main xanthone derivative (about 78\%) in mangosteen pericarp extract to be one of the major candidate compounds used as anti-breast cancer. The mechanism of $\alpha$-mangostin as an anti-cancer is as anti-proliferative associated with tumor growth suppression in vivo and metastasis in breast cancer model rat and inhibit the growth of breast cancer cells MCF-7 through decreased function of hERa receptors (most common breast cancer subtype). ${ }^{9} \alpha$-mangostin can also induces apoptosis of cancer cells through mitochondrial pathways, cell cycle retention through induction of $\mathrm{p} 21^{\mathrm{cip} 1}$ and Akt dephosphorylation on breast cancer cells, and inhibits invasion also migration of cancer cells in the breast gland. $\alpha$-mangostin showed anti-proliferative activity against MC-7 adenocarcinoma cell apoptosis with $\mathrm{IC}_{50}$ value of $20 \mu \mathrm{M} .{ }^{10-11}$ That $\mathrm{IC}_{50}$ value is classified as an active cytotoxicity category $(10-100 \mu \mathrm{M})^{12}$ but it can be increased. Therefore, it is necessary to increase the activity of $\alpha$-mangostin and its affinity as antagonist of estrogen receptor alpha through the computer-aided drug design (CADD) methodologies, such as molecular docking method and 3D structure-based pharmacophore modeling, were explored in this study.

Pharmacophore is a molecular framework defined as an essential part of a compound responsible for biological activity. Ligandscout Advanced is one of the most frequently used software in 3D modeling of pharmacophore from the protein-ligand complex. The software can provide information on $3 \mathrm{D}$ chemical structures covering the hydrophobicity, electrophilicity, donor, and hydrogen bond acceptor regions. ${ }^{13}$

Molecular docking is a computational method that can provide information about intermolecular interactions of proteins, nucleic acids, lipids, and ligands. The purpose of molecular docking is to obtain optimized 


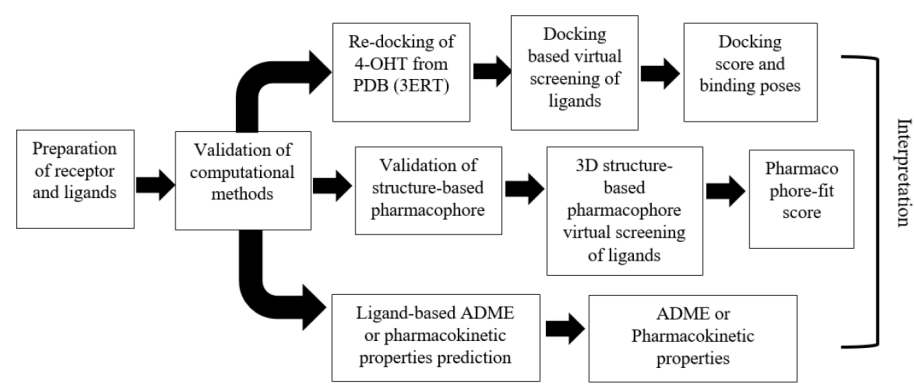

Figure 1: The scheme of methods.

conformation of proteins and ligands also obtain a relative orientation between proteins and ligands through the minimized energy free system. ${ }^{14}$

\section{METHODS}

The scheme of methods in this research is presented in Figure 1.

\section{Molecular Docking Simulation}

The receptor is the X-ray crystallography derived ERa in complex with 4-hydroxytamoxifen (4-OHT) downloaded from Protein Data Bank with PDB ID: 3ERT. ${ }^{15}$ The ligand was separated from receptor structures using BIOVIA Discovery Studio 2017 R2 Client. The 3D structures of a-mangostin and its derivatives as ligand were optimized by ChemOffice 2010 and ChemDraw Ultra 12.0 (PerkinElmer Inc.), also LigandScout 4.1 (Inte:Ligand $\mathrm{GmbH}$ ). The molecular docking simulations has been done according to a previously validation study. ${ }^{16}$ The ERa receptor and ligands were prepared for docking simulation using AutoDockTools 1.5.6. The receptor and ligands were protonated. The receptor as macromolecule has added the Kollman charges while the ligands have added the Gasteiger charges ${ }^{17}$ The grid parameter file is according to the grid box that comprised of $40 \times 40 \times 40$ points with $0.375 \AA$ space and was centered on the active site of $\mathrm{ER} \alpha(\mathrm{x}=30.010, \mathrm{y}=-1.913$, and $\mathrm{z}=24.207)$. AutoDock 4.2 (The Scripps Research Institute) was used to do the molecular docking simulation. The docking parameter file is according to Lamarckian Genetic Algorithm (LGA) with: 100 number of runs, 150 population size, 2.500 .000 energy evaluation, 0.02 rate of gene mutation, and 0.8 rate of crossover. ${ }^{18}$ The conformation results from the docking simulation were clustered using a root mean square deviation (RMSD) tolerance of $1.0 \AA$. The ligand conformation with the lowest free binding energy $(\Delta G)$ was chosen from the most favored cluster. The best ligand conformation was used for the further step of analysis. The receptor-ligand complexes from docking simulation were visualized using EduPyMOL 1.7 and BIOVIA Discovery Studio Visualizer 2017. The determination of ligand interaction features for each pose within the binding pocket of receptor were analyzed by LigandScout Advanced 4.1 Inte:Ligand $\mathrm{GmbH}$, Vienna, Austria. ${ }^{19}$

\section{D Structure-based Pharmacophore Modeling}

The 3D structure-based-pharmacophore model was derived from the $\mathrm{X}$-ray structure of ER $\alpha$ that complexed with 4-OHT (PDB ID: 3ERT) using Ligandscout 4.1 Advanced. ${ }^{20}$ The validation of 3D structure-based interaction feature model was done by screening the 626 actives set and 20,773 decoys set that obtained from the Database of Useful Decoys. ${ }^{21}$ The a-mangostin and its derivatives were screened virtually using the validated 3D structure-based pharmacophore model using LigandScout 4.1 Advanced algorithm. The results of this process are the pharmacophore-fit scores. The pharmacophore-fit score measured the similarity of features and geometry of each 3D structure-based hit compounds to the pharmacophore model features with 4 number of omitted features

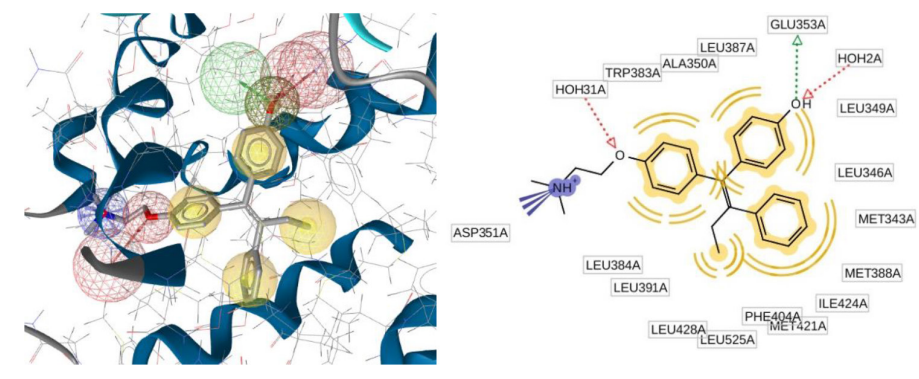

Figure 2: (a) 3D structure-based pharmacophore modeling of 4-OHT with ERa (PDB ID: 3ERT). Positive ionizable, hydrophobic, hydrogen bond donor and acceptor interactions are represented as blue star, yellow spheres, green and red arrows, respectively. (b) 2D structure-based 3ERT shown the hydrophobic interactions with the binding pocket residues.

for merged pharmacophore, $10.0 \%$ partially matching features optional threshold, and 1.0 feature tolerance scale factor.

\section{Ligand-based ADME or Pharmacokinetic Predictions of a-mangostin and Its Derivatives}

Pharmacokinetic properties of $\alpha$-mangostin and its derivatives were analyzed by the QikProp 4.2 module of Schrodinger Software Suites. ${ }^{22}$ Predicted ADME properties including the permeability through MDCK Cells (QPPMDCK), predicted gut-blood barrier (QPPCaco), and predicted $\log \mathrm{IC}_{50}$ score for blockage of $\mathrm{K}^{+}$channels (QPlogHERG), predicted aqueous solubility (QPlogS), predicted binding to human serum albumin (QPlogKhsa), and percentage of human oral absorption.

\section{RESULT}

The selected receptor for molecular docking simulation was the $\mathrm{x}$-ray structure of ERa that complexed with 4-OHT (PDB ID: 3ERT) based on a good experimental resolution (1.9 $\AA$ ), R-value free (0.262), and R-value work (0.229). ${ }^{15}$ The hydrophobic interaction on the 4 -OHT was predominantly interacted with aromatic rings and butenyl group also formed a positive ionizable interaction with secondary amine nitrogen. The hydrogen bond interactions were formed with the hydroxyl and phenoxy oxygens as shown in Figure 2. The ERa has ligand-binding domain ( $\mathrm{LBD}$ ) which is predominantly the hydrophobic cavity that composed by amino acid residues from helices $3,6,7,8,11$, and 12 . The agonist and antagonist activity of a ligand is determined by the helix-12 from residues 536-544 in its macromolecule (ERa). When an antagonist for example 4-OHT binds to LBD of ERa, the helix-12 will be closed and not binds to co-activator so it has the antagonist activity based on the absence of hydrogen bond interaction with His524. ${ }^{16}$ Whereas, estradiol as an agonist of ERa has the hydrogen bond interaction with His524. The validation of molecular docking simulation was done through the separation of 4-OHT from ERa structure and re-docking it into the binding pocket of ERa again. The best docked ligand (4-OHT) conformation had a root mean square deviation (RMSD) of $1.01 \AA$ compared to the original receptor structure conformation as shown in Figure 3.

\section{The Molecular Docking Simulation of Alpha Mangostin and Its Derivatives}

The best docked conformation of $\alpha$-mangostin within the ligand binding domain of ER $\alpha$ showed the hydrogen bond with $I$ and 3 hydroxy groups while the carbonyl group didn't form any interactions as shown in Figure 4

The predicted best binding pose comparison of 4-OHT and a-mangostin illustrated that one aromatic ring from each ligand is occupied the ligand binding domain of ERa in a similar way as shown in Figure 5. 


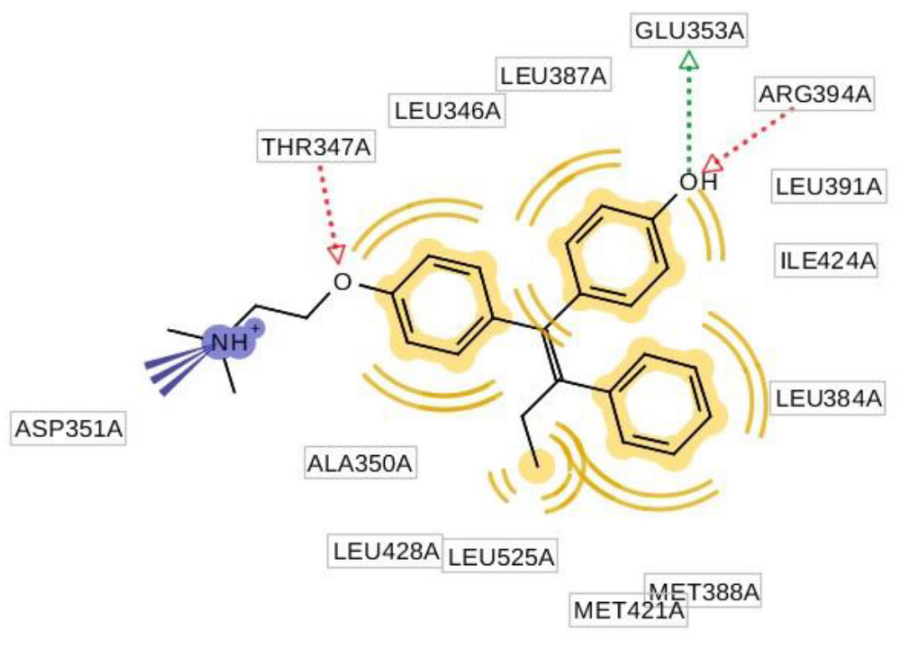

MET343A

Figure 3: The best docked pose of 4-OHT with ERa that was performed by AutoDock 4.2.
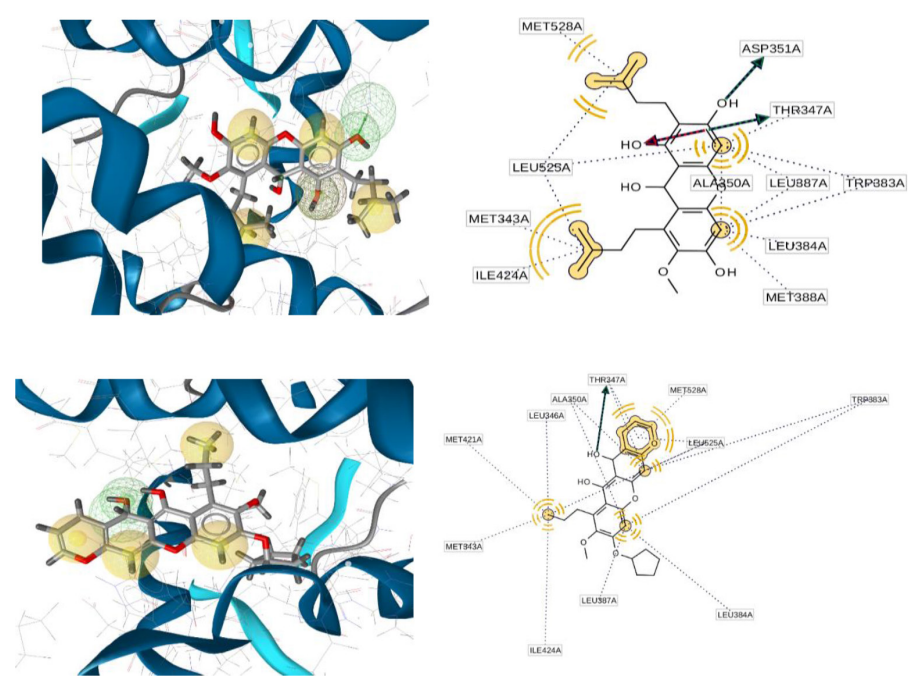

Figure 4: 3D and 2D structure-based pharmacophore modeling of the best docked pose of ERa that shows the hydrophobic interactions with binding pocket residues of (a) a-mangostin and (b) AMD10.

Figure 4 showed that the dimethylaminoethoxy group of 4-hydroxy tamoxifen (4-OHT) more extended than the methoxy group and hydroxy group of $a$-mangostin. The difference could be caused by the lower free binding energy $(\Delta \mathrm{G})$ of $4-\mathrm{OHT}(-11.40 \mathrm{kcal} / \mathrm{mol})$ compared to $\Delta \mathrm{G}$ of $\alpha$-mangostin $(-9.05 \mathrm{kcal} / \mathrm{mol})$. The design of new a-mangostin derivatives structure was focused on modification at methoxy group and dihydroxy-subtituted aromatic ring and based on the key interactions between 4-OHT and ERa as shown in Table 1 also based on Lipinski's Rule of Five and ERa as shown in Table 1 also based on Lipinski's Rule of Five as shown in Table 2

The free binding energy $(\Delta \mathrm{G})$ of $\alpha$-mangostin derivatives were ranged from -11.89 to $-9.37 \mathrm{kcal} / \mathrm{mol}$ compared to the $\alpha$-mangostin $(-9.05$ $\mathrm{kcal} / \mathrm{mol}$ ). The lowest $\Delta \mathrm{G}$ was AMD10 (- $11.89 \mathrm{kcal} / \mathrm{mol})$, lowest

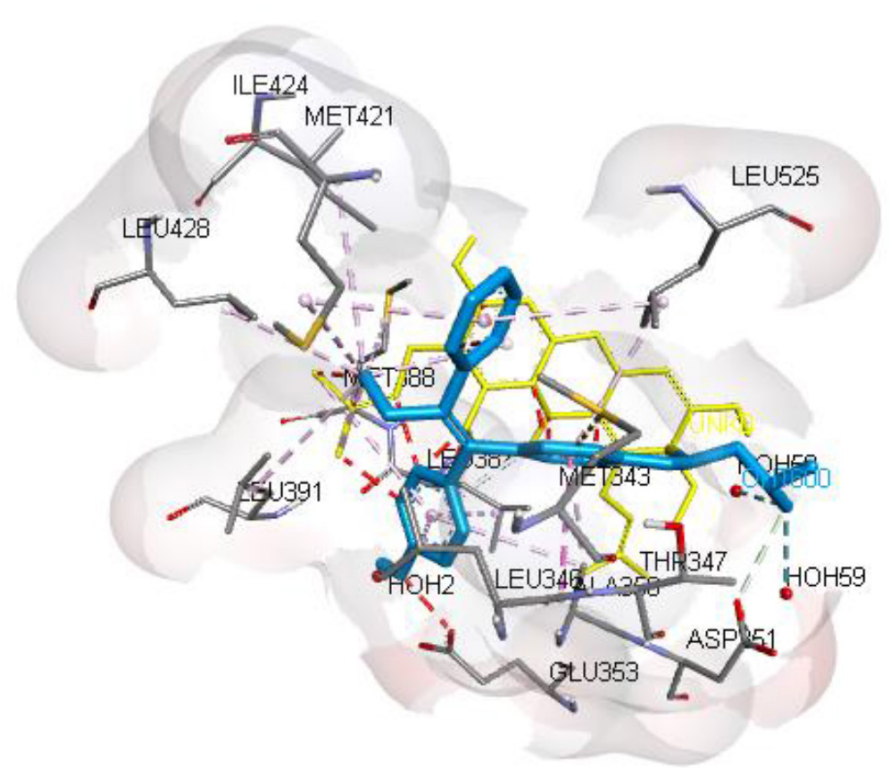

Figure 5: The overlay of the best docked pose of a-mangostin (yellow) and 4-OHT (light blue) in the binding pocket of ERa. Van der Waals interactions, hydrogen bonds, and pi-alkyl interactions are depicted as green, blue, and pink colored lines, respectively.

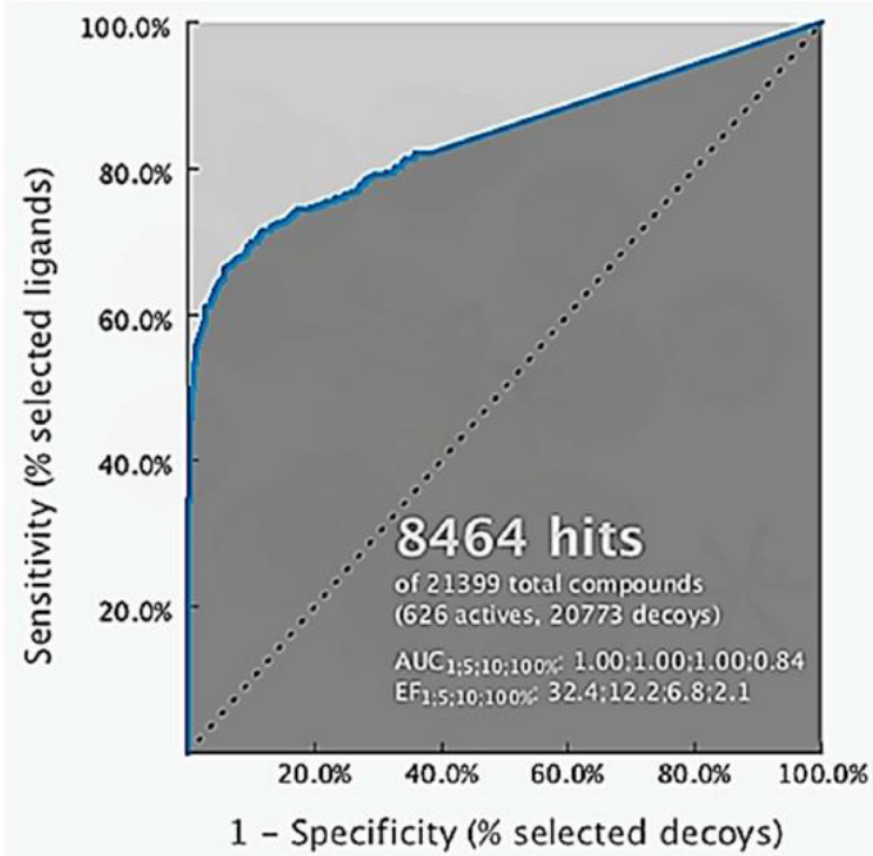

Figure 6: ROC validation curve of pharmacophore model.

calculated $\mathrm{Ki}(1.93 \mathrm{nM})$, and higher amount of clusters (94 clusters) than a-mangostin (65 clusters).

The 3D Structure-Based Pharmacophore Modeling of Alpha Mangostin and Its Derivatives

The validation of 3D structure-based pharmacophore and interaction features model was done by screening the 626 actives set and 20,773 decoys set that obtained from the Database of Useful Decoys (DUDe). ${ }^{21}$ The results showed the enrichment factor $100 \%\left(\mathrm{EF}_{100 \%}\right)$ was 32.4 with $\mathrm{AUC100 \%}$ was 1.00 as shown in Receiver Operating Characteristic 
Table 1: Derivatives of 1,3,6-trihydroxy-7-methoxy-2,8-bis(3-methylbut-2-enyl)xanthen-9-one (a-mangostin).

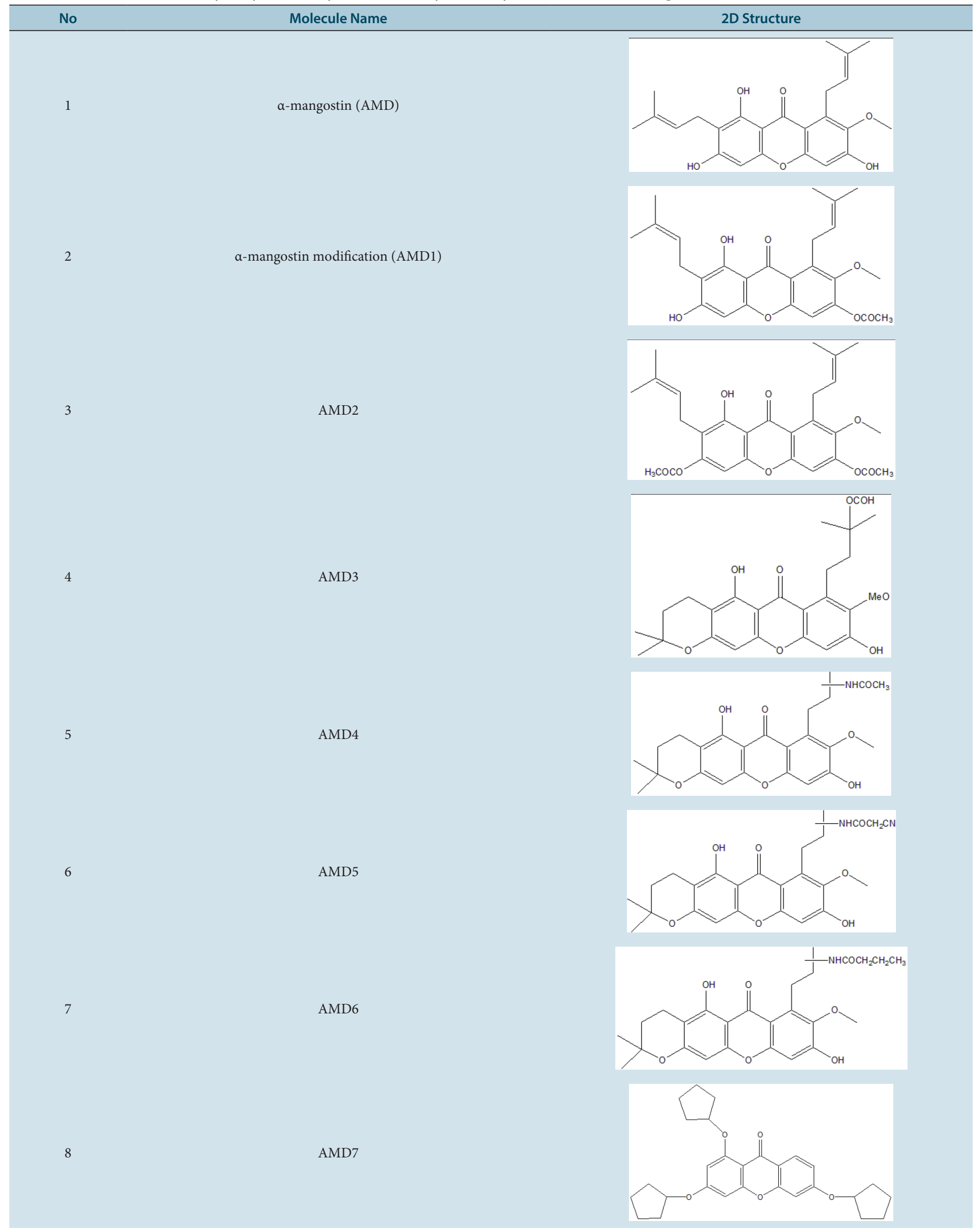


AMD8
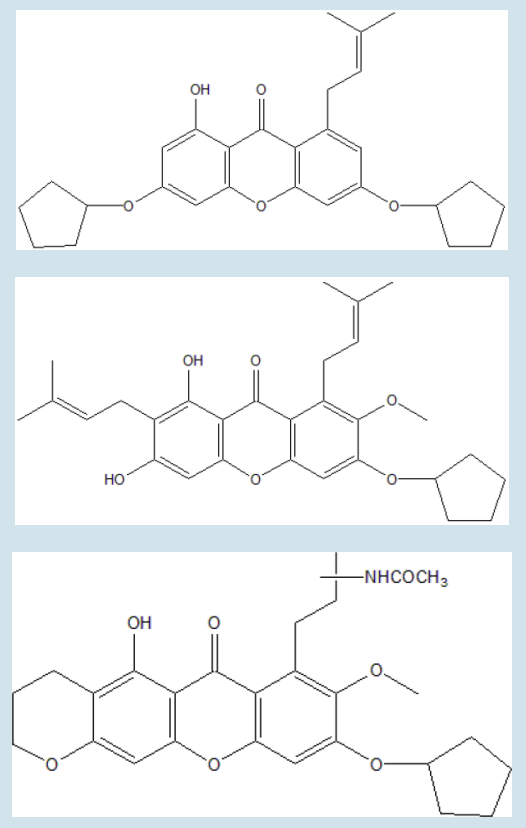

Table 2: Computed properties of a-mangostin and its derivatives based on Lipinski's Rule of Five.

\begin{tabular}{cccccc}
\hline No & Molecule Name & Molecular Weight & Log P & Number of Hydrogen Bond Donors & Number of Hydrogen Bond Acceptors \\
\hline 1 & a-mangostin & 418.530 & 3.708 & 2 & 6 \\
2 & AMD1 & 460.567 & 3.928 & 1 & 7 \\
3 & AMD2 & 498.620 & 4.290 & 1 & 7 \\
4 & AMD3 & 459.515 & 3.952 & 2 & 7 \\
5 & AMD4 & 458.531 & 4.181 & 4 & 5 \\
6 & AMD5 & 483.541 & 3.931 & 4 & 6 \\
7 & AMD6 & 486.585 & 4.817 & 4 & 5 \\
8 & AMD7 & 456.623 & 4.965 & 1 & 5 \\
9 & AMD8 & 456.623 & 4.092 & 1 & 5 \\
10 & AMD9 & 470.694 & 4.443 & 1 & 4 \\
11 & AMD10 & 493.556 & 4.740 & 3 & 4 \\
\hline
\end{tabular}

(ROC) curve (Figure 6). These results indicated that the 3D pharmacophore model was able to differentiate the active molecules from the decoy molecules.

The pharmacophore fit-score of $\alpha$-mangostin and its derivatives are reported in Table 4. The pharmacophore fit-score is the measurement of geometric similarity of the features of a molecule compared to $3 \mathrm{D}$ pharmacophore model. The results showed that $\alpha$-mangostin and AMD10 had a high pharmacophore-fit (83.06 and 86.46, respectively) which means the chemical features of $\alpha$-mangostin and AMD10 were well aligned geometrically to the 4-OHT chemical features.

\section{The Interpretation of Molecular Docking Simulation and 3D Pharmacophore Modeling}

As shown in Table 3, The results of docking simulation showed that the free binding energy $(\Delta \mathrm{G})$ of $\alpha$-mangostin and AMD10 were $-9.05 \mathrm{kcal} /$ $\mathrm{mol}$ and $-11.89 \mathrm{kcal} / \mathrm{mol}$, respectively which is $\Delta \mathrm{G}$ AMD10 is lower than tamoxifen $(-11.40 \mathrm{kcal} / \mathrm{mol})$. $\alpha$-mangostin formed two hydrogen bonds with Thr347 and Asp351, and eight hydrophobic interactions with Met388, Met528, Ile424, Arg394, Leu384, Leu387, Leu428, and Glu353 (Figure 7a), while AMD10 formed three hydrogen bonds with Thr347, Met343, and Met421 and twelve hydrophobic interactions with Asp351, Arg394, Glu353, Glu419, Met388, Met528, Ile424, Trp383, His524, Gly420, Phe404, and Leu349 (Figure 7b). The hydrophobic interactions with Leu384, Trp383, Leu349, and Phe404 were contributed essentially to the binding of a-mangostin and AMD10 compared to hydrophobic interactions of 4-OHT within LBD of ERa.

Van der Waals interactions, hydrogen bonds, and pi-alkyl interactions are depicted as green, blue, and pink colored lines, respectively. The results of 3D structure-based modeling showed that the 4-hydroxyl group on aromatic ring and 19, 20 methyl groups were hindered the complete mapping with the hydrophobic features (yellow spheres) of 4-OHT as shown in Figure 8. Whereas, the modified aromatic ring and 6 hydroxyl group on aromatic ring of AMD10 have a better alignment with the hydrophobic features of 4-OHT, so it produced a higher pharmacophore-fit score.

Ligand-based ADME or Pharmacokinetic Predictions of a-mangostin 
Table 3: The docking simulation results of a-mangostin and its derivatives in ligand binding domain of ERa.

\begin{tabular}{|c|c|c|c|c|c|c|c|}
\hline \multirow{2}{*}{ No } & \multirow{2}{*}{ Molecule Name } & \multirow{2}{*}{ Chemical Formula } & \multirow{2}{*}{$\begin{array}{c}\Delta \mathrm{G} \mathrm{kcal} / \\
\mathrm{mol}\end{array}$} & \multirow{2}{*}{$\begin{array}{l}\text { Number in } \\
\text { Cluster }\end{array}$} & \multirow{2}{*}{$\begin{array}{l}\text { Calculated Ki } \\
\qquad(\mathrm{nM})\end{array}$} & \multicolumn{2}{|c|}{ Interactions with Amino Acids } \\
\hline & & & & & & Hydrogen Bond & van der Waals (Hydrophobic) \\
\hline 1 & a-mangostin & $\mathrm{C}_{24} \mathrm{H}_{34} \mathrm{O}_{6}$ & -9.05 & 65 & 233.82 & Thr347, Asp351 & $\begin{array}{l}\text { Met388,Met528, Ile424,Arg394, } \\
\text { Leu384,Leu387, Leu428, Glu353 }\end{array}$ \\
\hline 2 & AMD1 & $\mathrm{C}_{26} \mathrm{H}_{36} \mathrm{O}_{7}$ & -9.70 & 76 & 78.00 & $\begin{array}{l}\text { Thr347, Asp351, } \\
\text { Met343 }\end{array}$ & $\begin{array}{c}\text { Cys530,Leu349,Leu384,Leu387,Phe4 } \\
\text { 04,Val533 }\end{array}$ \\
\hline 3 & AMD2 & $\mathrm{C}_{28} \mathrm{H}_{40} \mathrm{O}_{8}$ & -9.87 & 80 & 57.95 & Thr347 & $\begin{array}{c}\text { Gly420,Gly521,Leu346,Leu391,Leu428 } \\
\text {,Met388,Phe404,Trp383 }\end{array}$ \\
\hline 4 & AMD3 & $\mathrm{C}_{25} \mathrm{H}_{31} \mathrm{O}_{8}$ & -9.37 & 60 & 135.30 & Thr347, Asp351 & Met343,Met528,Lys529,Leu536 \\
\hline 5 & AMD4 & $\mathrm{C}_{25} \mathrm{H}_{32} \mathrm{~N}_{1} \mathrm{O}_{7}$ & -10.34 & 98 & 26.51 & Met421 & $\begin{array}{c}\text { Asp351,Glu353,Leu391,Met388,Met52 } \\
\text { 8,Phe404,Trp383,Val418 }\end{array}$ \\
\hline 6 & AMD5 & $\mathrm{C}_{26} \mathrm{H}_{31} \mathrm{~N}_{2} \mathrm{O}_{7}$ & -9.90 & 63 & 55.23 & $\begin{array}{l}\text { Thr347, } \\
\text { Met343 }\end{array}$ & $\begin{array}{l}\text { Asp351,Met528,Met421,Ile424, } \\
\text { Leu428,Leu346,Trp383,Val533 }\end{array}$ \\
\hline 7 & AMD6 & $\mathrm{C}_{27} \mathrm{H}_{36} \mathrm{~N}_{1} \mathrm{O}_{7}$ & -10.63 & 96 & 16.22 & $\begin{array}{l}\text { Thr347 } \\
\text { Met421 }\end{array}$ & $\begin{array}{c}\text { Arg394,Asp351,Glu353,Leu349,Leu3 } \\
\text { 84,Trp383 }\end{array}$ \\
\hline 8 & AMD7 & $\mathrm{C}_{28} \mathrm{H}_{40} \mathrm{O}_{5}$ & -11.41 & 65 & 4.35 & Asp351, Met343 & $\begin{array}{c}\text { Arg394,Gly521,His524,Met388,Leu3 } \\
\text { 49,Thr347 }\end{array}$ \\
\hline 9 & AMD8 & $\mathrm{C}_{28} \mathrm{H}_{40} \mathrm{O}_{5}$ & -10.79 & 86 & 12.26 & Thr347, Met343 & $\begin{array}{c}\text { Arg394,Asp351,Gly420,Glu353,Leu3 } \\
\text { 84,Met528 }\end{array}$ \\
\hline 10 & AMD9 & $\mathrm{C}_{29} \mathrm{H}_{40} \mathrm{O}_{4}$ & -11.11 & 90 & 7.14 & $\begin{array}{c}\text { Thr347, } \\
\text { Asp351, Met343 }\end{array}$ & $\begin{array}{c}\text { Arg394,Glu353, Glu419,Gly420,Phe4 } \\
\text { 04,Trp383 }\end{array}$ \\
\hline 11 & AMD10 & $\mathrm{C}_{28} \mathrm{H}_{31} \mathrm{~N}_{1} \mathrm{O}_{7}$ & -11.89 & 94 & 1.93 & $\begin{array}{l}\text { Thr347, Met343, } \\
\text { Met } 421\end{array}$ & $\begin{array}{l}\text { Asp351,Arg394,Glu353,Glu419 } \\
\text {,Met388,Met528,Ile424,Trp383, } \\
\text { His524,Gly420,Phe404,Leu349 }\end{array}$ \\
\hline
\end{tabular}

Table 4: The pharmacophore fit-score of $a$-mangostin and its derivatives.

\begin{tabular}{cccc}
\hline No & Molecule Name & $\begin{array}{c}\text { Pharmacophore-Fit } \\
\text { Score }\end{array}$ & $\begin{array}{c}\text { Docking Score } \\
\text { (kcal/mol) }\end{array}$ \\
\hline 1 & a-mangostin & 83.06 & -9.05 \\
2 & AMD1 & 63.27 & -9.70 \\
3 & AMD2 & 63.30 & -9.87 \\
4 & AMD3 & 96.28 & -9.37 \\
5 & AMD4 & 81.04 & -10.34 \\
6 & AMD5 & 71.65 & -9.90 \\
7 & AMD6 & 62.96 & -10.63 \\
8 & AMD7 & 58.52 & -11.41 \\
9 & AMD8 & 48.22 & -10.79 \\
10 & AMD9 & 68.10 & -11.11 \\
11 & AMD10 & 86.46 & -11.89 \\
\hline
\end{tabular}

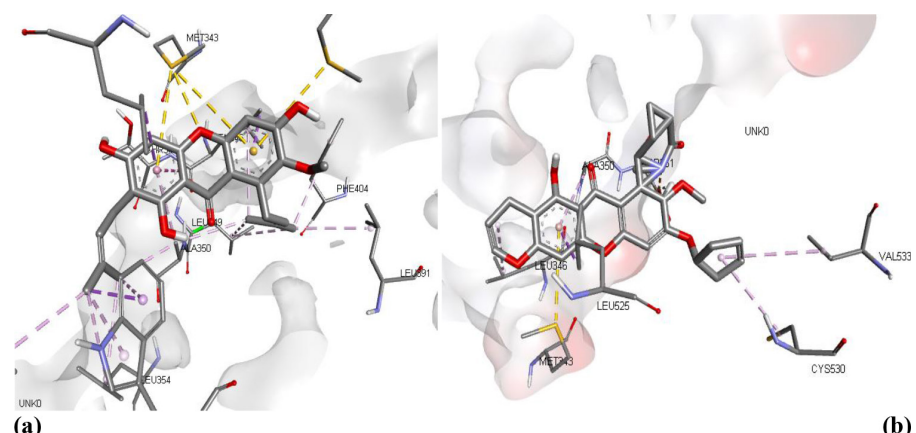

Figure 7: The interactions of a-mangostin (a) and AMD10 (b) within the ligand binding domain.

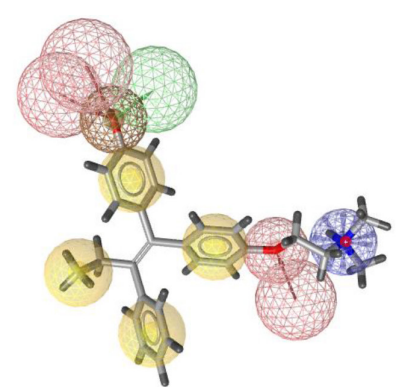

(a)

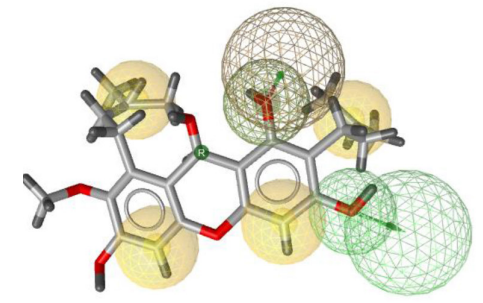

(b)

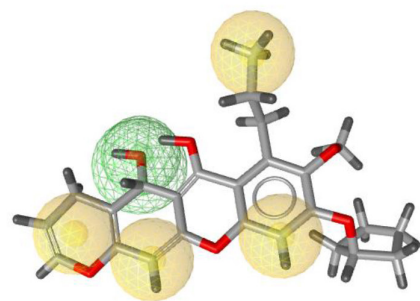

(c)
Figure 8: Fit of (b) a-mangostin and (c) AMD10 to the 3D structure based pharmacophore model derived from 4-OHT (a) with ERa (PDB ID: 3ERT). The 3D pharmacophore models were produced using LigandScout 4.1 Advanced. Positive ionizable, hydrophobic, hydrogen bond donor and acceptor interactions are represented as blue star, yellow spheres, green and red arrows (spheres), respectively. 
Muchtaridi, et al:: Prediction of Alpha Mangostin and Its Derivatives against Estrogen Receptor Alpha

Table 5: Ligand-based ADME or pharmacokinetic predictions of a-mangostin and its derivatives.

\begin{tabular}{|c|c|c|c|c|c|c|c|}
\hline Molecule & aQPlogHERG & ${ }^{\text {bQPPCaco }}$ & ${ }^{c} \mathrm{QP} \log B \mathrm{~B}$ & ${ }^{\mathrm{d}} \mathrm{QPPMDCK}$ & eQPlogS & ${ }^{\text {fQPlogKhsa }}$ & $\begin{array}{l}{ }^{g} \text { (percent human) } \\
\text { Oral Absoption }\end{array}$ \\
\hline a-mangostin & -2.929 & 1146.076 & -0.888 & 573.262 & -2.334 & -0.297 & 93.044 \\
\hline AMD2 & -3.950 & 1100.608 & -1.268 & 548.719 & -2.814 & -0.459 & 81.186 \\
\hline AMD3 & -3.144 & 849.678 & -0.964 & 414.842 & -2.710 & -0.280 & 90.025 \\
\hline AMD6 & -5.034 & 245.408 & -0.728 & 119.887 & -2.243 & -0.426 & 76.687 \\
\hline AMD7 & -3.800 & 7037.655 & -0.000 & 4076.799 & -4.598 & 0.371 & 100.000 \\
\hline AMD8 & -3.861 & 3953.998 & -0.361 & 2186.129 & -4.564 & 0.380 & 100.000 \\
\hline AMD9 & -3.798 & 2385.921 & -0.747 & 1266.344 & -3.955 & 0.161 & 100.000 \\
\hline
\end{tabular}

(a) Predicted $\mathrm{IC}_{50}$ score for blockage of HERG K+ channels (Acceptable range limit - above : 5.0); (b) Predicted Caco 2 cell permeability in nm/sec ( $\leq 25$ : poor; $\geq 500$ : great), (c) Predicted brain/blood partition coefficient (Acceptable range -3.0 to 1.0); (d) Predicted MDCK cell permeability in nm/sec ( $\leq 25$ : poor; $\geq 500$ : great); (e) Predicted aqueous solubility in mol/L (Acceptable range -6.5 to 0.5); (f) Predicted binding to human serum albumin (Acceptable range -1.5 to 1.2 ); (g) Percentage of human oral absorption $\left(\geq 80 \%\right.$ : high)..$^{22}$

\section{and Its Derivatives}

In addition, analysis of pharmacokinetic profiles of $\alpha$-mangostin and its derivatives were analyzed to identify the acceptable range defined for human use as shown in Table 5.

\section{DISCUSSION}

The $\mathrm{x}$-ray structure of ERa that complexed with 4-OHT (PDB ID: 3ERT) was selected based on a good experimental resolution (1.9 $\AA$ ), R-value free $(0.262)$, and R-value work (0.229). ${ }^{15}$ The $\mathrm{R}$-value work is used to assess the progress in refinement model of X-ray crystallographic data, and also can be used as a factor in evaluating the quality of a molecular crystal model. R-value work is a measure of error between the observed intensity of the diffraction pattern and the predicted intensity calculated from the model. R-value free is a quantity of statistics to assess the model quality of X-ray crystallographic data. It is calculated the same way as the $R$ value, but from subset of data set aside for $R$-value free calculation, and not used in model refinement. As a rule of thumb, models with $\mathrm{R}$ values that far exceed (resolution / 10) should be handled with care. Thus, if the resolution of a model is $2.5 \AA$, the model of R-value free should not exceed $0.25 .^{16}$

The ligand binding domain (LBD) of ERa is predominantly the hydrophobic cavity that composed by amino acid residues of helix-12. Helix-12 is composed by His524 and when an antagonist ligand binds to LBD of ERa, the helix-12 will be closed and not binds to co-activator. ${ }^{16}$ The agonist and antagonist activity of the ligand is determined by this helix 12 of the 536-544 residues in its macromolecule (hER $\alpha$ ). When a 4-OHT antagonist binds to a hERa LBD, the helix-12 will be closed and not attached to the co-activator so as to have antagonistic activity based on the absence of hydrogen bonding interactions with His524. While estradiol as a hER $\alpha$ agonist has a hydrogen bonding interaction with His524. ${ }^{22}$

The design of the a-mangostin derivatives was focused on the modification of methoxy groups and dihydroxy substituted aromatic rings, 3-methylbut-2-enyl groups, and also based on the principal interaction between 4-OHT and hERa. The design of structural modification also considers the Lipinski rule or known as Lipinski’s Rule of Five regarding the active compound administered orally and this rule establishes four physicochemical parameters (molecular weight $\leq 500, \log \mathrm{P} \leq 5$, donor hydrogen bond $\leq 5$, and acceptor hydrogen bond $\leq 10$ ) associated with $90 \%$ of the active drug administered orally that has reached clinical phase II. These physicochemical parameters relate to acceptable solubility and permeability of the intestinal tract and are part of the early stages that determine oral bioavailability. ${ }^{22}$

The structure of the hERa protein has a hydrogen bond on its constituent amino acid residues that is between Glu419 with His524 and Glu419 with Lys531 (hydrogen bond network). The disturbance of this hydrogen bonds network can be represented by fluctuations by a ligand has potential as an antagonist against hER $\alpha$ receptors. ${ }^{16}$ The $\alpha$-mangostin and AMD10 ligands disrupt the hydrogen bonds by fluctuations with Ser432 and Ser521 residues thus it could be clarified that $\alpha$-mangostin and AMD10 might have antagonistic activity against hER $\alpha$.

a-mangostin and AMD10 had not formed the hydrogen bond interaction with His524, thus a-mangostin and AMD10 are potentially as an antagonist agent. The estrogen-like agonist effects of 4-OHT in the uterus is determined by the distance of ligand interaction to Asp351. Shorter distance to Asp351 decreased the agonist activity of 4-OHT in uterus. ${ }^{6}$ The distance of ligand interaction to Asp351 was measured and compared. The results showed the distances of the 4-OHT, a-mangostin, and AMD10 to Asp351 were $3.20 \AA$, $2.21 \AA$, and $2.54 \AA$, respectively, These results indicated that $\alpha$-mangostin and AMD10 could form stronger interactions with Asp351 residue and potentially decreased the estrogenlike agonist effects of 4-OHT in the uterus.

Pharmacophore modeling can determine the fit score of pharmacophore features against $\alpha$-mangostin and structural modifications. The pharmacophore fit score is a percentage of geometric similarity measure of chemical features compared with the active $3 \mathrm{D}$ model of pharmacophore ligand ie tamoxifen. ${ }^{16}$ The results showed that $\alpha$-mangostin and AMD10 have high pharmacophore compatibility values $(\geq 50 \%)$ of $83.06 \%$ and $86.46 \%$, respectively, which means geometrically similar chemical features of a-mangostin and AMD10 with chemical features 4-OHT. It might be concluded that $\alpha$-mangostin and AMD10 have a good affinity for hER $\alpha$. In addition, the ADME or pharmacokinetic properties were evaluated. The results showed that all the pharmacokinetic parameters of $\alpha$-mangostin and its derivatives were within the acceptable range defined for human use.

\section{CONCLUSION}

The essential interactions of $\alpha$-mangostin and its derivatives with the estrogen receptor alpha (ER $\alpha)$ consists the hydrogen bond and hydropho- 
bic interactions. AMD10 was the best derivatives among the all designed a-mangostin derivatives that was identified by molecular docking simulation and 3D structure-based pharmacophore modeling. a-mangostin, AMD10, and other derivatives are meet all of Lipinski's Rule of Five. The binding free energy $(\Delta \mathrm{G})$ of $\alpha$-mangostin and AMD10 were $-9.05 \mathrm{kcal} / \mathrm{mol}$ and $-11.89 \mathrm{kcal} / \mathrm{mol}$, respectively. The pharmacophorefit scores of alpha mangostin and AMD10 were $83.06 \%$ and $86.46 \%$, respectively. $\alpha$-mangostin and AMD10 are need the further pharmacological investigation and for future development of $\alpha$-mangostin and its best derivative as the novel of anti-breast cancer agent with antagonistic activity to ERa also better safety and side-effect profiles compared to tamoxifen.

\section{ACKNOWLEDGEMENT}

This study was supported by The Directorate General of Higher Education of The Ministry of Research and Technology of Indonesia.

\section{CONFLICT OF INTEREST}

The authors declare no conflict of interest.

\section{ABBREVIATIONS}

ADME: Absorption, Distribution, Metabolism, and Excretion; CADD: Computer-Aided Drug Design; ERa: Estrogen Receptor a; LBD: Ligand Binding Domain; LGA: Lamarckian Genetic Algorithm; OHT: Hydroxytamoxifen; PDB: Protein Data Bank; RMSD: Root Mean Square Deviation.

\section{SUMMARY}

The molecular interactions of alpha mangostin and its derivatives with estrogen receptor $\alpha(E R \alpha)$ were analyzed using molecular docking simulation and showed the overall binding energy of $\alpha$-mangostin derivatives have better affinity to ER $\alpha$ compared to its bacis structure. The binding energy of AMD10 as best alpha mangostin derivative was $-11.89 \mathrm{kcal} /$ mol compared to basic $\alpha$-mangostin $(-9.05 \mathrm{kcal} / \mathrm{mol})$. AMD10 formed the interactions with Thr347, Asp351, Met388, Met528, Ile424, Arg394, and Glu353. The pharmacophore-fit scores of basic alpha mangostin and AMD10 were $83.06 \%$ and $86.46 \%$, respectively. ADME properties were also predicted and met all the acceptable criterias. These results showed that AMD10 is promising candidate of novel anti-breast cancer agent.

\section{REFERENCES}

1. WHO. World Health Statistics 2017 : Monitoring Health for the SDG. Villars sous Yens: World Health Organization. 2017.

2. Ministry of Health of Indonesia. Data and Information on Indonesia Health Profile. Jakarta: Ministry of Health Republic of Indonesia. 2017.

3. Mijatovic T, Van Quaquebeke E, Delest B, Debeir O, Darro F, Kiss R. Cardiotonic steroids on the road to anti-cancer therapy. Biochim Biophys Acta. 2007: 1776:32-57

4. Hafiz H. Epigenetic Mechanisms of Tamoxifen Resistance in Luminal Breast Cancer. Diseases. 2017;5(3):1-11.

5. Bhatt S, Stender JD, Joshi S, Wu G, Katzenellenbogen BS. OCT-4: A Novel
Estrogen Receptor- $\alpha$ Collaborator That Promotes Tamoxifen Resistance in Breast Cancer Cells. Oncogene. 2016;35(44):5722-34.

6. Martinkovich S, Shah, D., Planey, S., Arnott, J Selective estrogen receptor modulators: tissue specificity and clinical utility. Clin Interv Aging. 2014;9(3):1437-52.

7. Malervaa G, Parkb J, Zouc L, Hub Y, Moradpoura Z, Pearlbergb J, et al. Highthroughput ectopic expression screen for tamoxifen resistance identifies an atypical kinase that blocks autophagy. PNAS. 2011;108(5):2058-63.

8. Lu R, Hu X, Zhou J, Sun J, Zhu A, Xu X, Zheng H. COPS5 amplification and overexpression confers tamoxifen-resistance in ER $\alpha$-positive breast cancer by degradation of NCoR. Nat Commun. 2016;7(12):1-13.

9. Shibata MA, Linuma M, Morimoto J, Kurose H, Akamatsu K, Okuno Y, et al. $\alpha$-Mangostin extracted from the pericarp of the mangosteen (Garcinia mangostana Linn) reduces tumor growth and lymph node metastasis in an immunocompetent xenograft model of metastatic mammary cancer carrying a p53 mutation. BMC Med. 2011;9(1):69-79

10. Setiawati A, Octa F, Riswanto D, Yuliani S. Anticancer Activity of Mangosteen Pericarp Dry Extract Against MCF-7 Breast Cancer Cell Line through Estrogen Receptor - $\alpha$. Indones J Pharm. 2014;25(3):119-24.

11. Kurose $H$, Shibata M, linuma M, Otsuki Y. Alterations in Cell Cycle and Induction of Apoptotic Cell Death in Breast Cancer Cells Treated with $\alpha$-Mangostin Extracted from Mangosteen Pericarp. J Biomed Biotechnol. 2012;6(7):1-9.

12. Weerapreeyakul N, Nonpunya A, Barusrux S, Thitimetharoch T. Evaluation of the anticancer potential of six herbs against a hepatoma cell line. Chin Med. 2012;7(15):1-7.

13. Wolber G, Langer T. Ligandscout: 3-D pharmacophores derived from proteinbound ligands and their use as virtual screening filters. J Chem Inf Model. 2005;45(1):160-9.

14. Ferreira L, DosSantos R, Oliva G, Andricopulo A. Molecular docking and structure-based drug design strategies. Molecules. 2015;20(2):13384-421.

15. Shiau AK, Barstad D, Loria PM, Cheng L, Kushner PJ, Agard DA, Greene GL. The structural basis of estrogen receptor/coactivator recognition and the antagonism of this interaction by tamoxifen. Cell. 1998;95(7):927-37.

16. Muchtaridi M, Yusuf M, Diantini A, Choi SB, Al-Najjar BO, Manurung JV, et al. Potential activity of fevicordin-a from Phaleria macrocarpa (Scheff) Boerl. seeds as estrogen receptor antagonist based on cytotoxicity and molecular modelling studies. Int J Mol Sci. 2014;15(5):7225-49.

17. Weiner SJ, Kollman PA, Case DA, Singh UC, Ghio C, Alagona G, et al. New Force Field for Molecular Mechanical Simulation of Nucleic Acids and Proteins. J Am Chem SOC. 1984;106(3):765-84.

18. Morris G, Huey R. AutoDock4 and Auto Dock Tools4: Automated docking with selective receptor flexibility. J Comput Chem. 2009;30(16):2785-91.

19. Muchtaridi M, Syahidah HN, Subarnas A, Yusuf M, Bryant SD, Langer T. Molecular Docking and 3D-Pharmacophore Modeling to Study the Interactions of Chalcone Derivatives with Estrogen Receptor Alpha. Pharmaceuticals. 2017;10(4):1-12.

20. Mysinger MM, Carchia M, Irwin JJ, Shoichet BK. Directory of useful decoys, enhanced (DUD-E): Better ligands and decoys for better benchmarking. J Med Chem. 2012;55(14):6582-94

21. Jorgensen WL, Duffy EM. Prediction of Drug Solubility from Structure. Adv Drug Deliv. Rev. 2002;54(3):355-66.

22. Ramachandran B, Kesavan, S., Rajkumar, T. Molecular modeling and docking of small molecule inhibitors against NEK2. Biomed Informatics. 2016;12(2):62-8.

23. Lipinski C. Lead Profiling Lead- and Drug-like Compounds: The Rule-of-Five Revolution. Drug Discov. Today. 2004;1(4):337-41.

Article History: Submission Date : 26-02-2018; Revised Date : 09-04-2018; Acceptance Date : 04-05-2018.

Cite this article: Muchtaridi M, Dermawan D, Yusuf M. Molecular Docking, 3D Structure-Based Pharmacophore Modeling, and ADME Prediction of Alpha Mangostin and Its Derivatives against Estrogen Receptor Alpha. J Young Pharm. 2018;10(3):252-9. 